\title{
HEART SOUNDS AND INTRACARDIAC PRESSURES IN MITRAL STENOSIS
}

\author{
BY \\ DESMOND JULIAN * AND L. G. DAVIES
}

From the National Heart Hospital and the Institute of Cardiology

Received January 10, 1957

It has been thought for many years that, in the absence of conduction defects, the time of occurrence of the heart sounds is largely determined by intracardiac pressure and flow changes. It is convenient to examine this hypothesis in mitral stenosis, where the exact time of mitral valve opening and closure can be obtained from the phonocardiogram. This usually shows two abnormal features (Wells, 1954) in addition to the diastolic murmur. First there is often an increase of the time between the QRS of the electrocardiogram and the onset of the maximal vibrations of the first heart sound (Weiss and Joachim, 1911; Cossio and Berconsky, 1943). These vibrations arise in the mitral valve and this interval may be called $R-\mathbf{M}_{1}$ if one measures from the onset of the intrinsicoid deflection as has been done in this study: other workers refer to a $\mathbf{Q}-\mathbf{M}_{1}$ interval, but for the purposes of measurement, we found the peak of $R$ better than $Q$. Presumably, mitral valve closure is delayed by the raised pressure in the left atrium and there should be a relation between this pressure and the $\mathrm{R}-\mathrm{M}_{1}$ : certainly as Kelly (1955) has pointed out, mitral stenosis is the only condition in which this interval is prolonged. One result of this delay is that the sound due to mitral valve closure becomes superimposed on that arising from tricuspid valve closure, and this effect contributes to the loud first sound in mitral stenosis.

The second abnormality is the presence of an added sound-the opening snap of the mitral valve-and since this is a left-sided event its time of occurrence is best measured from the instant of aortic valve closure. This measurement will be referred to as the A-OS interval and is the time taken for the left ventricular pressure to fall from that of aortic valve closure to the left atrial levelwhen the mitral valve opens. Again it seems reasonable to suppose that there should be a relation between this time and the left atrial pressure.

The suggestion that these two intervals were dependent on the pressure gradient across the mitral valve was made by Messer et al. (1951). Further work by Wells (1954) showed that the intervals could be correlated with the size of the mitral valve as measured at operation: this relationship must be indirect for the third variable, flow, was unknown; and his data are unsatisfactory in that he was unable to relate the time intervals with pressures recorded simultaneously. We have found important variations, particularly in the A-OS interval when comparing phonocardiograms taken at the time of cardiac catheterization and other times.

In this study the hypothesis relating sounds to pressures has been examined by correlating the $\mathrm{R}-\mathrm{M}_{1}$ and A-OS intervals with the coincident left atrial pressures. As already indicated we thought it essential that the phonocardiograms, electrocardiograms, and intracardiac pressures should be recorded simultaneously. We hoped that the intervals and pressures would in fact show a correlation close enough to predict the swing of left atrial pressure from measurement of the phonocardiogram; and further that this information could be used to estimate the degree of mitral valve obstruction without the necessity of cardiac catheterization, but this object was not

\footnotetext{
* Present address: Peter Bent Brigham Hospital, Boston, Mass.
} 
achieved because, as will be seen, the left atrial pressure was sometimes found to be less important than other factors in determining the $\mathrm{Q}-\mathrm{M}_{1}$ interval.

\section{METHODS}

Synchronous tracings of intracardiac pressures, phonocardiograms, and electrocardiograms were obtained on a N.E.P. photographic recorder from 27 patients with mitral valve disease. A fast paper speed of $80 \mathrm{~cm}$. a second was used to improve accuracy of measurement. The sternal angle was taken as the zero point of reference and the records were obtained during held expiration except where otherwise stated; Fig. 1, 2, and 3 illustrate the method in detail. The wedged pulmonary artery pressure was obtained at cardiac catheterization and we assumed that this is equivalent to the left atrial pressure pulse, though it

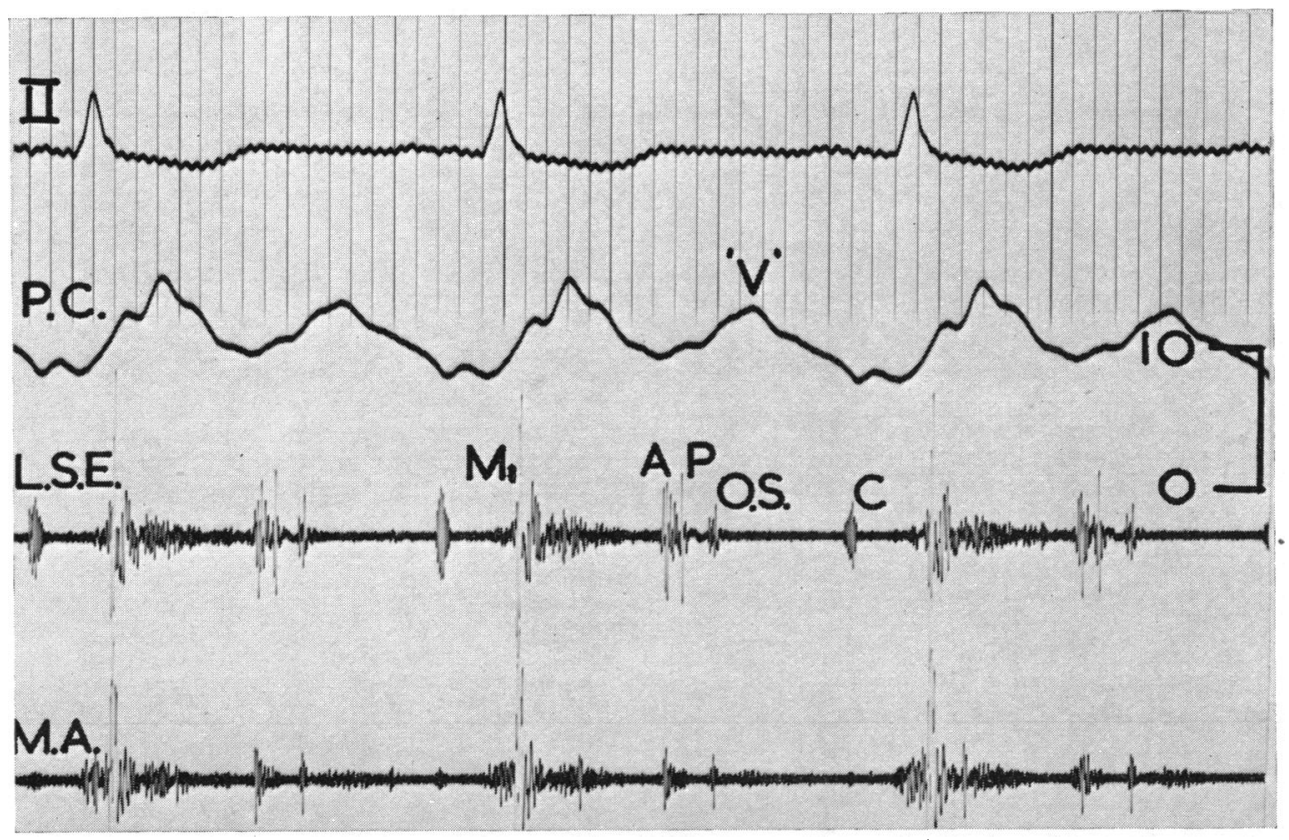

Fig. 1.-The method of obtaining the R-M and A-OS intervals together with the synchronous "left atrial "pressure. The small time intervals are $0.04 \mathrm{sec}$. and the calibration is in mm. of mercury. The peak of $v$ appears later than the opening snap because of a slight delay in the recording system. The peak of $a$ is distorted by the first heart sound artefact probably due to the transmission through the catheter of the impact of tricuspid valve closure.

$$
\begin{array}{lll}
\text { II--lead II of electrocardiogram } & \mathbf{M}_{1} \text { - mitral } \\
\text { P.C.-wedged pulmonary artery tracing } & \text { A } & \text { - aortic } \\
\text { L.S.E. phonocardiograms at left sternal } & \text { P } & \text { - pulmonary } \\
\text { M.A. J edge and mitral area } & \text { O.S. - mitral opening snap }
\end{array}
$$

is not less than $0.02 \mathrm{sec}$. later (Epps and Adler, 1953). In addition the pressure record is further delayed by the instrumental lag in the catheter and manometer system. For this reason the $v$ peak and the opening snap do not appear synchronous (Fig. 1). A further difficulty in the interpretation and measurement of the left atrial pressure pulse may arise as the result of artefacts occurring at the time of the first and second heart sounds (see Fig. 1, 2, and 3), artefacts that may be due to the transmission through the catheter of the impact of tricuspid and pulmonary valve closure. Because of these artefacts and further slight distortion due to the presence of $f$ waves (Fig. 3), the peak of $v$ may sometimes appear to precede the opening snap. This of course is impossible and the true pressure at $v$ has to be measured a little after the snap (usually $0.04-0.08 \mathrm{sec}$.). $z$ was taken as the nadir before the $c$ wave of the left atrial pressure pulse. 

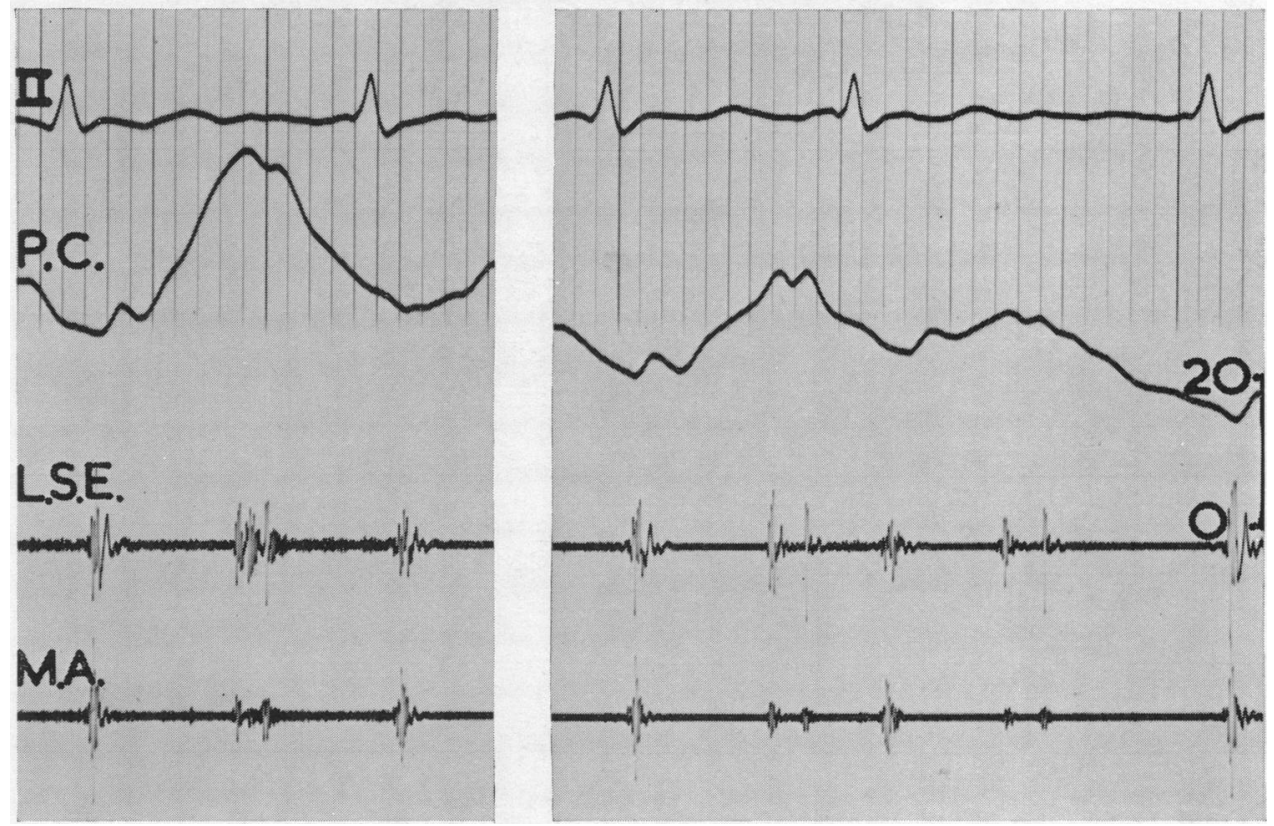

FIG. 2.-Tracings arranged as in Fig. 1. In this patient deep respiration was found to produce a marked swing in the "left atrial" pressure. It can be seen that a high pressure at $v$ produces a short A-OS interval and that this interval is prolonged when the pressure falls. (This is the same patient as in Fig. 5.)

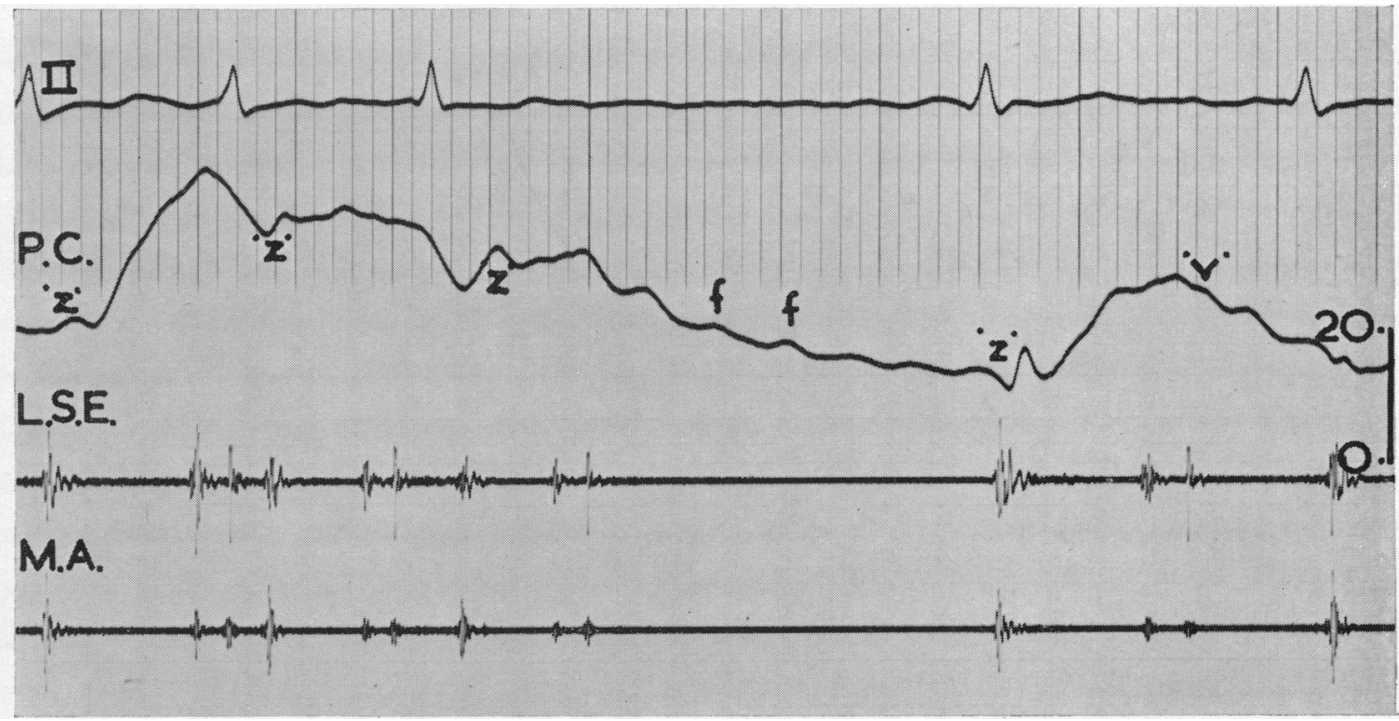

Fig. 3.-Tracings arranged as in Fig. 1. In this patient the effect of a long diastolic pause in shortening the $\mathbf{R}-\mathbf{M}_{1}$ interval and lengthening the $\mathrm{A}-\mathrm{OS}$ interval is well seen. These changes are clearly related to the synchronous " left atrial "pressures at $z$ and $v$. Artefacts at the time of the first heart sound are prominent and there is further distortion of the pressure tracing by fibrillation waves. 
In most of the patients a brachial artery tracing was obtained as part of the catheter procedure. A dicrotic notch could be identified in all and was taken as indicating the pressure at the time of aortic valve closure. Lead II of the cardiogram was recorded routinely and measurements of the $R-M_{1}$ interval were taken from the peak of the $R$ wave to the initial large vibration of the first heart sound.

High-frequency, logarithmic phonocardiograms (Leatham, 1952) were obtained with microphones placed at the cardiac apex and the fourth left interspace close to the sternum. In all records the mitral first sound, the aortic and pulmonary components of the second sound, and the opening snap were clearly distinguishable. In many of the records the tricuspid first sound could also be identified.

\section{RESULTS}

$R-M_{1}$ Interval Correlated with the Pressure Level at $\mathrm{z}$. The relationship between the $\mathrm{R}-\mathrm{M}_{1}$ interval and the level of pressure at $z$ is shown in Fig. 4. In 300 normal and abnormal subjects with conditions other than mitral stenosis, the $R-M_{1}$ interval did not exceed $0.045 \mathrm{sec}$. In half of our patients with mitral stenosis this interval was within this normal range. Even in the

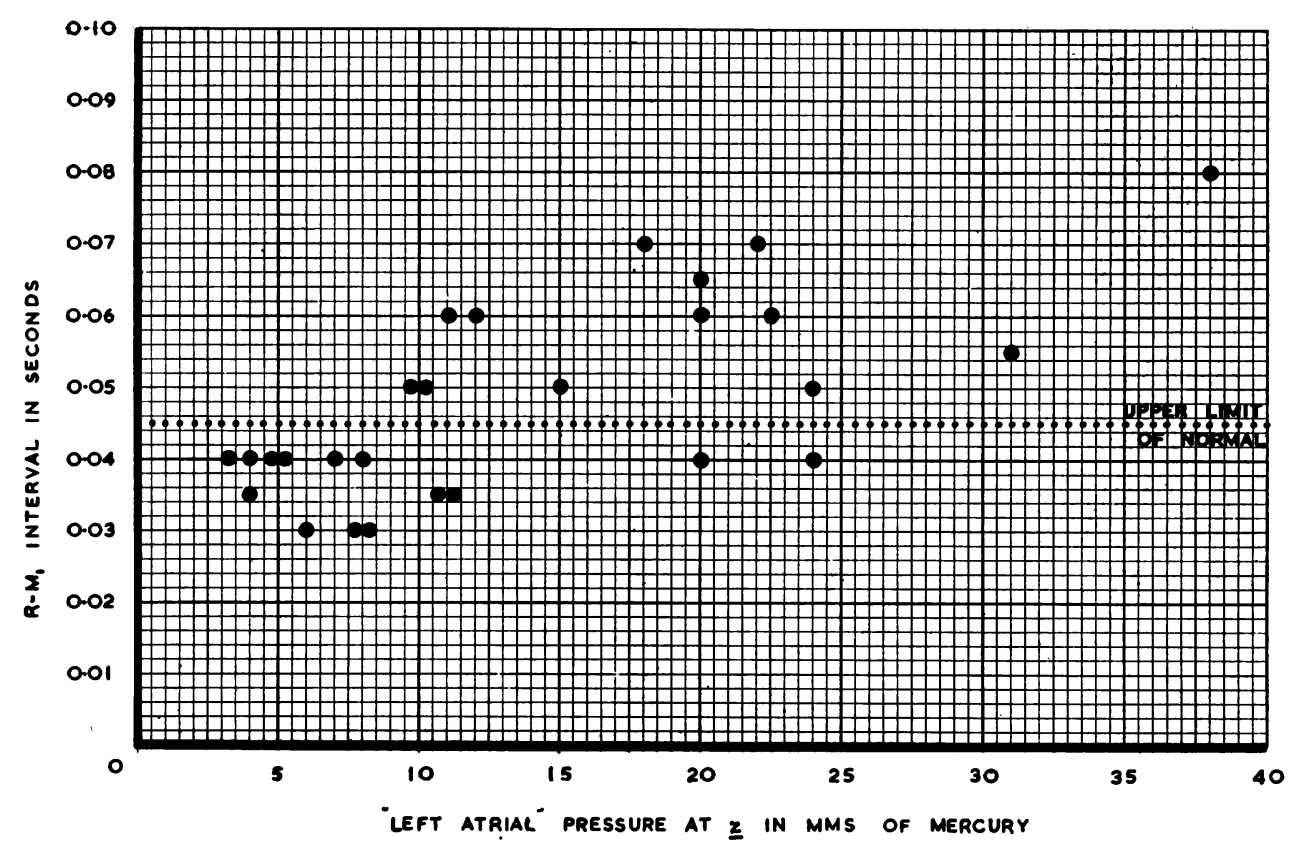

FIG. 4.-The relation between the $R-M_{1}$ interval and the simultaneous " left atrial " pressure (at $z$ ) in 27 patients with mitral valve disease.

remainder, where the $\mathbf{R}-\mathrm{M}_{1}$ interval was outside and often well outside normal limits, the correlation was poor. The results in two patients who showed wide variations in pressures and times have been recorded: in one (Fig. 5) the relation between the R-M interval and the pressure at $z$ was rather closer than the results in the whole group of patients (Fig. 4), but in the other there was no correlation at all (Fig. 6). Reasons for these discrepancies will be considered later.

A-OS Interval Correlated with the Pressure Level at $\mathrm{v}$. There was a fair correlation between the A-OS interval and the level of pressure at $v$ and this relationship appeared to be linear (Fig. 7). In the two patients already mentioned (Fig. 5 and 6) the correlation was much closer. Returning to the results as a whole (Fig. 7) it seemed probable that the scatter was due to differences in aortic pressure between one patient and another. For example if two patients have the same $v$ pressure, the A-OS interval should be longer in the one with the higher aortic pressure. This relationship is illustrated in Fig. 8, where the aortic valve closing pressure has been plotted as a hypothetical value of $v$ at which the A-OS interval would be zero. This value for each patient has been joined 


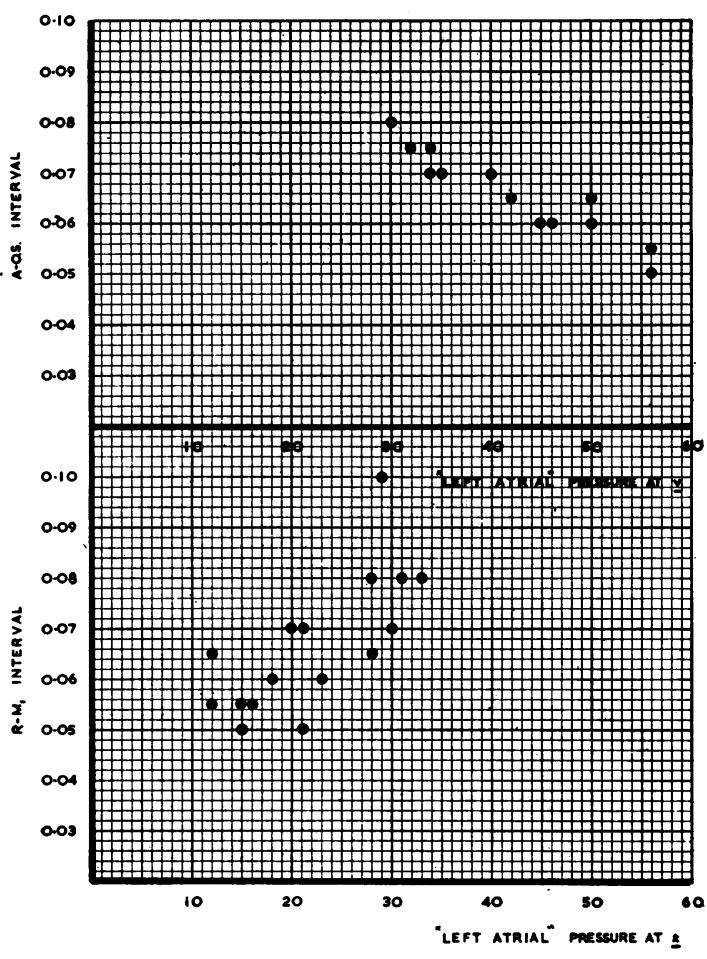

FIG. 5.-In this patient with mitral stenosis and auricular fibrillation, deep respiration produced a wide swing in "left atrial" pressure. There was a good correlation between the varying A-OS interval and the simultaneous pressure (at $v$ ). Unlike the patient in Fig. 6 , the $R-M_{1}$ interval did show some variation though the correlation with the simultaneous pressure (at $z$ ) was only fair.

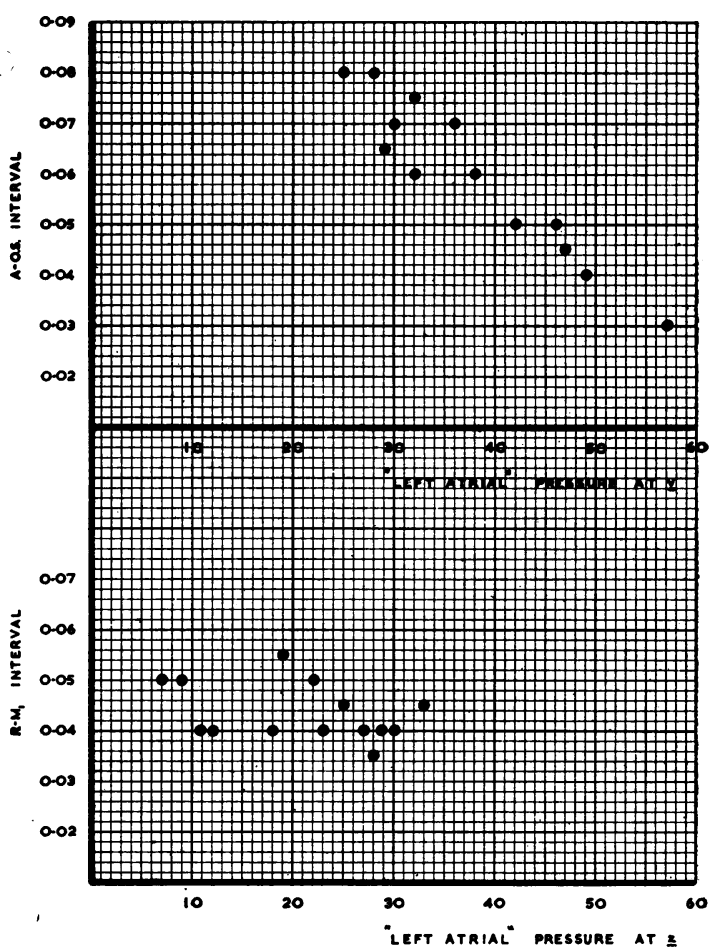

FIG. 6.-In this patient with mitral stenosis, aortic incompetence, and auricular fibrillation, exercise produced a sharp rise in the "left atrial" pressure. The A-OS interval shortened and there was a good correlation between this interval and the simultaneous pressure (at $v$ ). There was little change in the $\mathrm{R}-\mathrm{M}_{1}$ interval and no correlation between it and the rising "left atrial " pressure (at $z$ ).

to a second point relating the actual A-OS interval with the measured left atrial pressure at $v$. It is then clear that high aortic pressures do make the opening snap occur later and that low aortic pressures are associated with early opening snaps. The slope of the lines joining the paired points is an expression of the rate of isometric relaxation and in these patients showed little variation.

\section{Discussion}

$R-M_{1}$ Interval. It is now generally accepted that mitral valve closure produces the first, and tricuspid valve closure the second component of the first heart sound. The time of mitral valve closure as expressed by the $R-M_{1}$ interval could be influenced by the following factors: (1) the pressure gradient between the left atrium and left ventricle at the onset of ventricular systole, (2) the rate of rise of ventricular pressure, (3) the electrical-mechanical interval of the left ventricle, and (4) the pliability of the valve.

Other workers on this subject have thought that the height of the left atrial pressure is the chief factor in prolonging the R-M time (Messer et al., 1945; Kelly, 1955). Kelly points out that mitral stenosis is the only condition in which the $\mathbf{Q}-\mathbf{M}_{1}$ interval is prolonged. In mitral incompetence there is no gradient and this interval is normal. Kelly found, however, that only half his patients with mitral stenosis showed an increase of the $Q-M_{1}$ interval and that in some with tight mitral stenosis it remained within the normal range. These observations correspond with our experience (Fig. 4). If the $R-M_{1}$ interval was prolonged, then the coincident left atrial pressure was always 


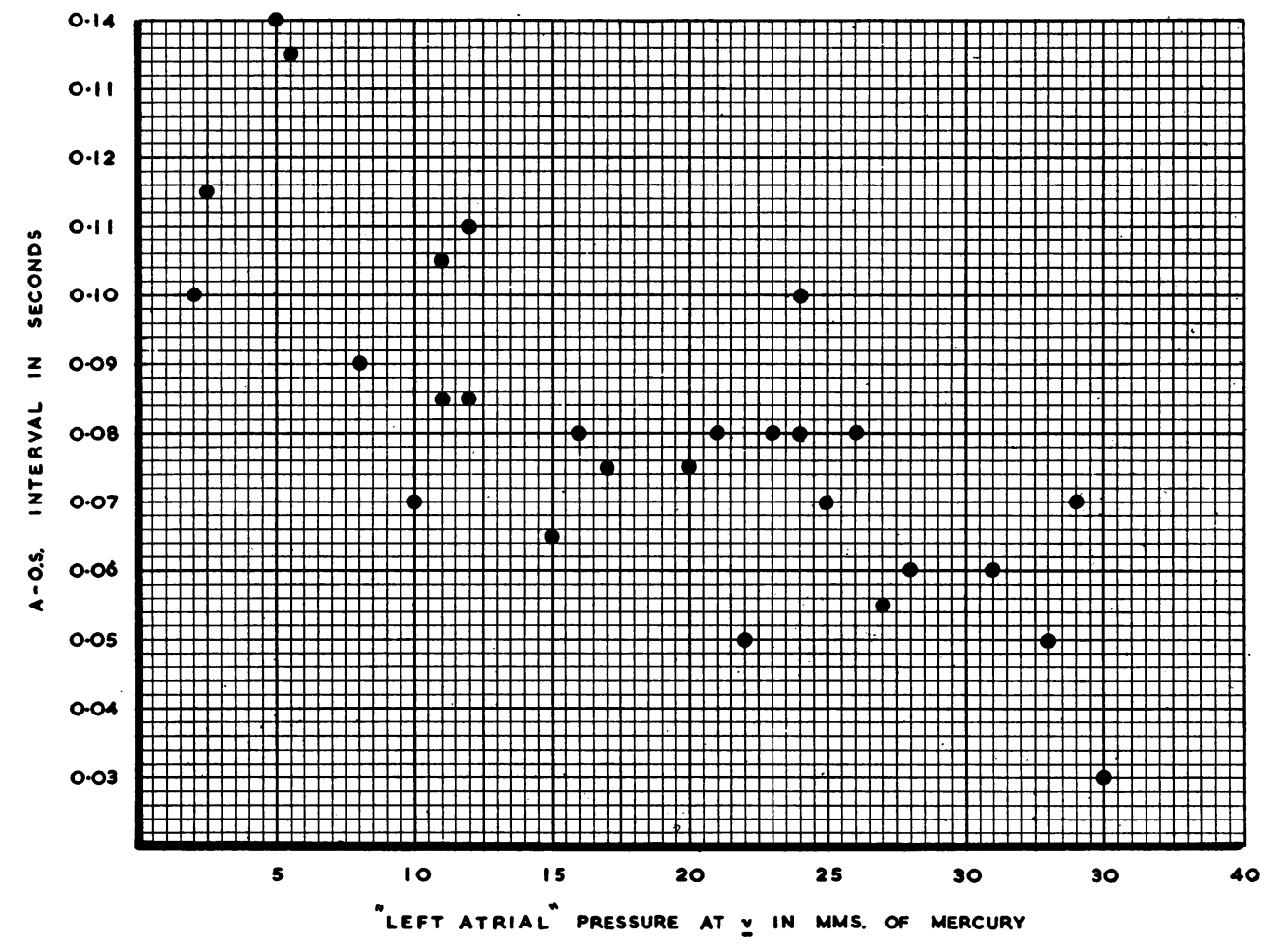

FIG. 7.-The relation between the A-OS interval and the simultaneous " left atrial" pressure (at $v$ ) in 27 patients with valve disease.

found to be raised. However, the converse does not hold and a rise of pressure may occur with no increase of this interval. The value of this measurement is therefore limited and it cannot be used to derive an index of the degree of mitral valve obstruction.

However, this discrepancy is of considerable interest, and the most satisfactory explanation of it is that the left ventricular pressure must rise more rapidly in some patients than in others. The rate of pressure rise when measured at operation varied between 4 and $14 \mathrm{~mm}$. $\mathrm{Hg}$ per $0.01 \mathrm{sec}$; this was in patients with mitral stenosis, but details of associated valve lesions were not given (Kelly, 1955). It seems reasonable to suppose that an aortic valve lesion would be the most likely cause of an increased rate of rise of left ventricular pressure, but it would have to be of more than trivial degree. Two outstanding examples of patients with a raised left atrial pressure $(z$ levels of 20 and $24 \mathrm{~mm} . \mathrm{Hg}$ ) and normal $\mathrm{R}-\mathrm{M}_{1}$ intervals both had important aortic incompetence (Fig. 4). One is the patient whose complete data are given in Fig. 6, and although the left atrial pressure varied widely it showed no correlation with the synchronous $\mathbf{R}-\mathrm{M}_{1}$ intervals. In contrast, in Fig. 5 there is a fair correlation between the $R-M_{1}$ intervals and simultaneous left atrial pressures and this patient had no aortic valve lesion.

Another possible explanation is that, if for any reason there was a rise in left ventricular diastolic pressure, the gradient between left ventricle and atrium would be decreased and this would shorten the $\mathbf{R}-\mathbf{M}_{1}$ interval. However, this state of affairs was unlikely to have been present in any of our patients. Another factor that might account for the discrepancy, though very little is known about it, is the electrical-mechanical interval of the left ventricle which has been reported (Kelly, 1955) to vary between 0.02 and $0.06 \mathrm{sec}$. This range is wide enough for the interval or delay to be a potential cause of substantial differences in the $R-M_{1}$ time.

Some workers (Wells, 1954; Nichols et al., 1955) have suggested that rigidity and calcification of 


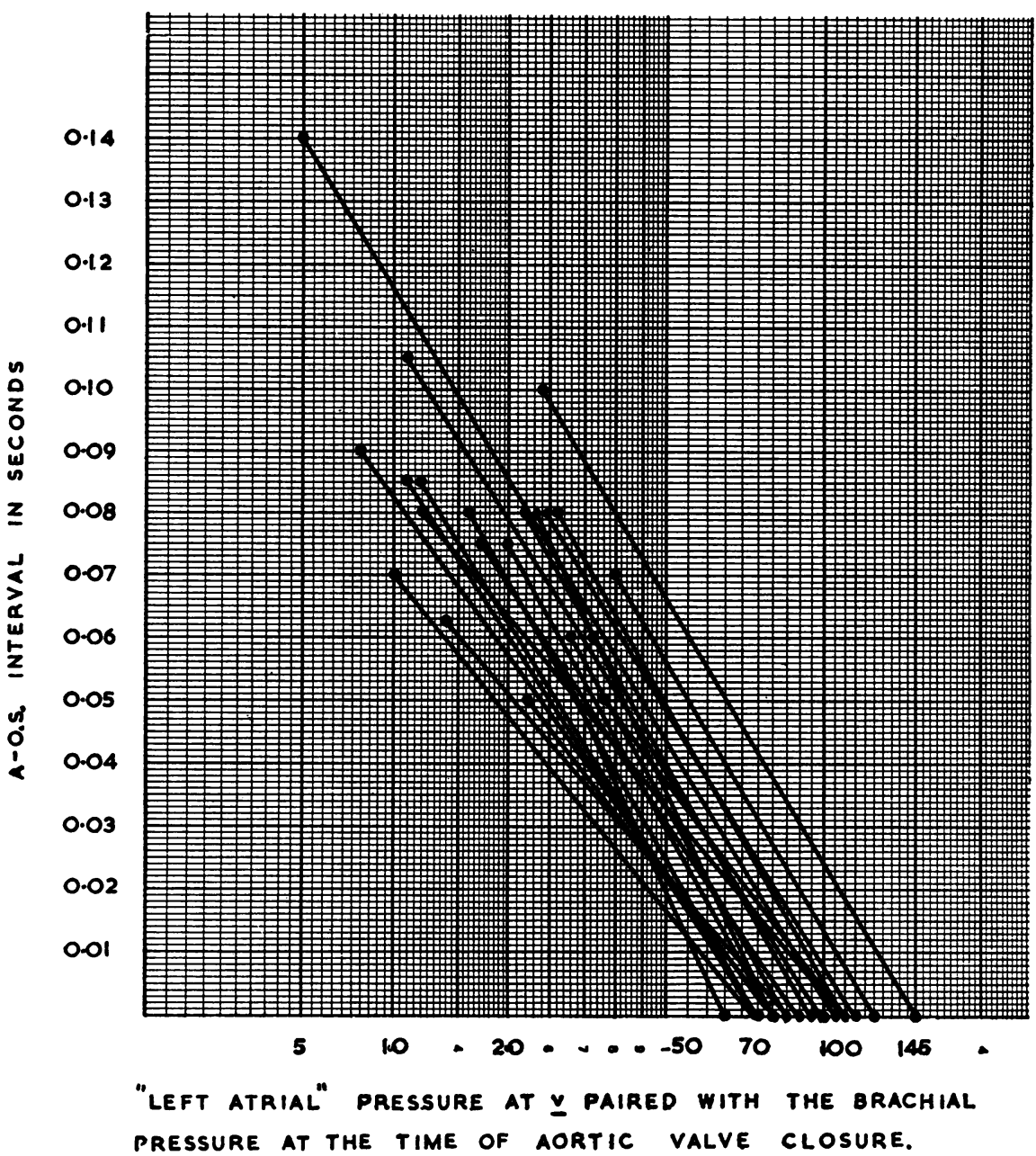

FIG. 8.-In mitral stenosis it is theoretically possible for the A-OS time to be zero; this state of affairs would require the "left atrial " pressure to equal the left ventricular pressure at aortic valve closure.

In each of these patients the aortic valve closing pressure has been taken from the brachial artery tracing and plotted as a hypothetical value of $v$. Each value has been joined to a second point which relates the actual A-OS time with the measured pressure level at $v$. The slope of the paired points is fairly constant and illustrates how the time of occurrence of the opening snap partly depends on the aortic pressure.

the mitral valve might cause delay in closure and lead to a prolonged $Q-M_{1}$ time. This seems improbable; most patients with mitral incompetence have rigid valves but an increase of the Q- $\mathbf{M}_{1}$ interval is not met with in this condition. Further, if such changes delayed mitral valve closure they should also delay mitral valve opening but, as will be seen, there is no evidence of this.

There are, therefore, several factors that might influence the relation between the $R-\mathbf{M}_{1}$ interval and the coincident left atrial pressure. They limit the value of the phonocardiogram as an indication of this pressure.

$A-O S$ Interval. It would seem probable that the following factors could influence the A-OS interval: (1) the height of pressure in the left atrium, and (2) the height of pressure in the left ventricle at the end of systole (i.e. aortic valve closure pressure). 
The importance of the left atrial pressure in determining the time of mitral valve opening is most simply illustrated when there is auricular fibrillation. Margolies and Wolferth (1932) noted that the snap was earlier following a short diastolic period than after one long enough to allow more complete emptying of the left atrium with a corresponding fall in pressure. This mechanism is illustrated in Fig. 3. The timing of the opening snap is accepted as giving evidence of the height of the coincident left atrial pressure and our findings show that this relationship is a linear one. There are, however, two points of practical importance that require emphasis. The first is that the A-OS interval should be correlated with the pressure at $v$ and not the mean capillary venous pressure as was done by Bayer et al. (1956). It is true that in mitral stenosis, both $v$ and $z$ frequently approximate to the mean and therefore both $v$ and the mean may correlate well with the A-OS interval. But in mitral incompetence where there is a steep $v$ wave this will not be so. In our group of 27 patients, there were four who had some incompetence in addition to stenosis and a further two where the incompetence was dominant. In these six, the relation between the left atrial pressure at $v$ and the A-OS interval was similar to that in the others with pure mitral stenosis. It follows that when a mixed lesion is present, an early snap is not reliable evidence of a raised mean left atrial pressure.

The second point is that the A-OS interval and the left atrial pressure at $v$ are not a direct index of the size of the mitral valve even when there is no incompetence; and this is because of another variable, the cardiac output. With moderately severe mitral stenosis and a large flow, the pressure at $v$ will be high and the A-OS interval short. With the same sized valve the findings will be very different with a small flow. This point may also be illustrated at the bedside where the A-OS interval can often be heard to vary with respiration, for in the presence of tight mitral stenosis, the increased pulmonary flow resulting from inspiration raises the left atrial pressure and the snap becomes earlier.

The other factor that is known to influence the timing of the opening snap is the aortic pressure. Bayer et al. (1955) were able to lengthen the A-OS interval by raising the systemic pressure with nor-adrenalin, the left atrial pressure remaining unaltered. Our findings confirm the importance of this factor and in Fig. 8 it will be seen that high aortic pressures make the opening snap later and low aortic pressures make it earlier.

Although variations in the rate of rise of left ventricular pressure have been reported there is little information about the rate of fall of pressure. Our results show indirectly that in our patients there was little variation and this factor may be of no importance in determining the time of mitral valve opening. When the systemic pressure has been taken into account, the correlation between the A-OS interval and the simultaneous left atrial pressure is close enough for us to believe that no further factors are involved. There is thus no reason to suppose that changes in the rigidity of the cusps are of any importance in determining the time of occurrence of the opening snap.

\section{SUMMARY}

Twenty-seven patients with mitral valve disease were studied by synchronous cardiac catheterization, phonocardiography, and electrocardiography with the object of examining the relationship between intracardiac pressures and the times of occurrence of the first heart sound and opening snap.

The $\mathrm{R}-\mathrm{M}_{1}$ interval (from the onset of the intrinsicoid deflection to the onset of the maximal vibrations of the first heart sound) was within normal limits in half these patients, even though the simultaneous left atrial pressure (at $z$ ) was sometimes raised. In the remainder the $\mathbf{R}-\mathbf{M}_{1}$ interval was increased but the correlation between interval and pressure was poor. These discrepancies are probably due to differences in the rate of rise of left ventricular pressure, and a rapid pressure rise shortens the $\mathbf{R}-\mathbf{M}_{1}$ interval back towards normal limits. Such a rise could result from associated aortic valve lesions which were found in the discrepant cases. The value of the measurement in predicting the simultaneous left atrial pressure is thus limited; however, it always appeared to be raised when the $\mathbf{R}-\mathbf{M}_{\mathbf{1}}$ interval was prolonged. 
The time of occurrence of the opening snap, expressed as the A-OS interval (from the onset of the first component of aortic valve closure to the opening snap) showed a fair correlation with the coincident left atrial pressure (at $v$ ). This relationship, which appeared to be linear, was also dependent on the level of pressure in the left ventricle at the time of aortic valve closure. High aortic pressures make the opening snap later and low aortic pressures make it earlier.

We wish to express our thanks to Dr. Paul Wood for his advice and criticism.

\section{REFERENCES}

Bayer, O., Loogen, F., and Wolter, H. H. (1956). Amer. Heart J., 51, 234.

Cossio, P., and Berconsky, I. (1943). Rev. Argent. Cardiol., 10, 162.

Epps,R. G., and Adler, R. H. (1953). Brit. Heart J., 15, 298.

Kelly, J. J. (1955). Amer. J. Med., 19, 862.

Leatham, A. (1952). Brit. med. Bull., 8, 333.

Margolies, A., and Wolferth, C. C. (1932). Amer. Heart J., 7, 443.

Messer, A. L., Counihan, T. B., Rappaport, M. B., and Sprague, H. B. (1951). Circulation, 4, 576.

Nichols, H. T., Likoff, W., Goldberg, H., and Lisan, P. 1955). Amer. Heart J., $50,577$.

Weiss, O., and Joachim, G. (1911). Ztschr. klin. Med., 73, 240.

Wells, B. (1954). Brit. Heart J., 16, 261. 